\title{
Efecto Zeeman Normal
}

Normal Zeeman Effect

Recibido: agosto 12 de 2015 | Revisado: setiembre 14 de 2015 | Aceptado: octubre 9 de 2015

Carlos Calderón Chamochumbi ${ }^{1}$

1 Facultad de Ingeniería y Arquitectura, Universidad de San Martín de Porres

calderonc@usmp.pe

\section{Abstract}

The Normal Zeeman Effect is described and a general derivation of the torque undergone by a magnetic dipole due to its interaction with an external magnetic field is presented here. The corresponding calculations of the differential element of potential magnetic energy and total magnetic energy are standard.

Key words: derivation, torque, dipole, magnetic field

\section{Resumen}

Se describe el Efecto Zeeman Normal y se presenta una derivación general del torque experimentado por un dipolo magnético debido a su interacción con un campo magnético externo. Los cálculos correspondientes al elemento diferencial de energía potencial magnética y de la energía potencial magnética convencional son estándares.

Palabras clave: derivación, torque, dipolo, campo magnético 


\section{Introducción}

En el curso de Física III, Física Moderna, asignado al $5^{\circ}$ Ciclo Académico del Plan de Estudios de la Escuela Profesional de Ingeniería Electrónica se estudia, entre muchos otros tópicos, el Efecto Zeeman Normal. Este fenómeno físico consiste en el desdoblamiento de las líneas espectrales atómicas al devenir energía potencial magnética causada por la interacción del momento dipolar magnético del propio átomo con un campo magnético externo constante, pero de dirección arbitraria (Belser, 1988). Se hace necesario calcular, en primer lugar, el torque experimentado por el dipolo atómico debido al campo magnético externo. En segundo lugar, se deduce el elemento diferencial de energía potencial magnética creado y, finalmente, se obtiene la energía potencial magnética total convencional. En la mayoría de las fuentes de referencia, se da una derivación ad hoc del torque por lo que aquí se presenta un tratamiento general. Los cálculos correspondientes al elemento diferencial de energía potencial magnética creada y a la energía potencial magnética total convencional son estándares.

\section{Desarrollo}

Un dipolo magnético atómico es una pequeñísima espira circunferencial a ser ubicada en el plano $\mathrm{z}=0$, de radio a, que porta una corriente I que circula en el sentido del acimut $\phi y$, por tanto, genera su propio campo magnético. Se adscribe al dipolo magnético un momento dipolar magnético $\mu$ cuyo módulo $\mu$ está definido como el producto del valor de la corriente por el área de la espira, es decir, $\mu=\mathrm{I} \pi \mathrm{a}^{2}$, y cuyo vector normal unitario apunta en la dirección del pulgar al enroscar los demás dedos de la mano derecha en el sentido de la circulación de la corriente, vale decir, k. Para calcular el torque, $d \tau$, causado al dipolo magnético $\mu=\mathbf{k} \mathrm{I} \pi \mathrm{a}^{2}$ por su interacción con el campo magnético uniforme $\mathbf{B}_{0}=\mathrm{B}_{0}\left(\mathbf{i} \operatorname{Sen} \theta_{0} \operatorname{Cos} \varphi_{0}+\mathbf{j} \operatorname{Sen} \theta_{0} \operatorname{Cos} \varphi_{0}\right.$ $\left.+\mathbf{k} \operatorname{Cos} \theta_{0}\right)$ en el que $B_{0}$ es su módulo, considérese la geometría mostrada en la Figura 1.

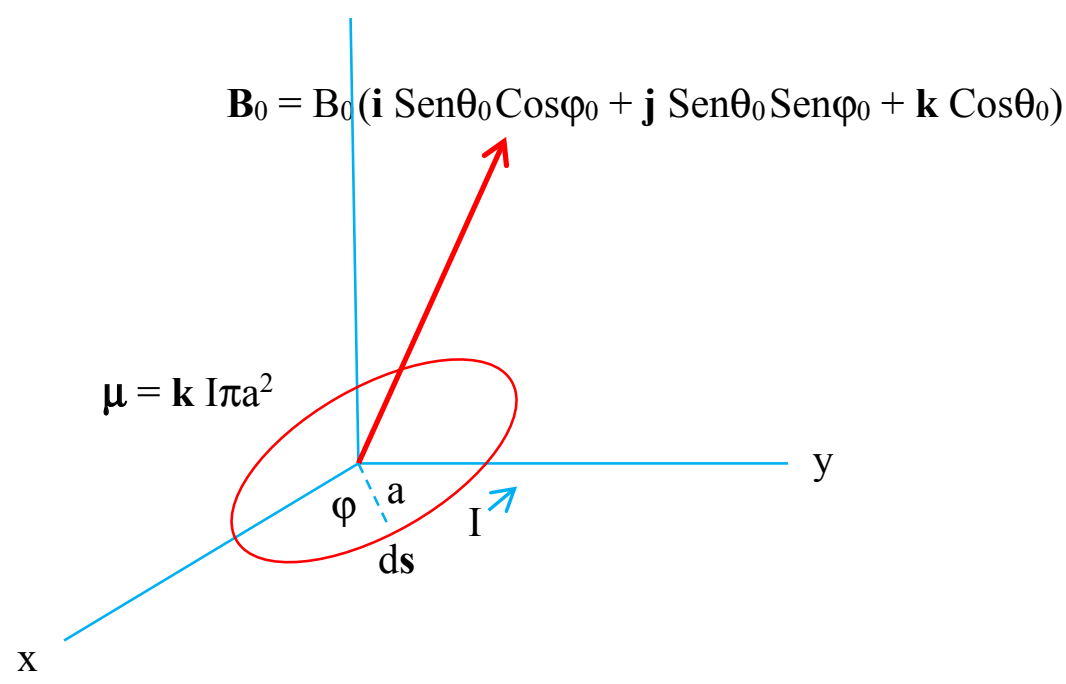

Figura 1. Geometría utilizada para el cálculo del torque $\tau$ causado al dipolo magnético $\mu=\mathbf{k}$ $\mathrm{I} \pi \mathrm{a}^{2}$ por su interacción con un campo magnético $\mathbf{B}_{0}=\mathrm{B}_{0}\left(\mathbf{i} \operatorname{Sen} \theta_{0} \operatorname{Cos} \varphi_{0}+\mathbf{j} \operatorname{Sen} \theta_{0} \operatorname{Cos} \varphi_{0}+\mathbf{k}\right.$ $\left.\operatorname{Cos} \theta_{0}\right)$.

La fuerza diferencial dF sobre el elemento diferencial de corriente I ds viene dada por la ley de la fuerza magnética de Lorentz:

$$
\mathrm{d} \mathbf{F}=\mathrm{I} \mathrm{d} \mathbf{s} \times \mathbf{B}_{\mathbf{0}}
$$

Debido a que ds y el radio vector $\mathbf{r}=\mathrm{r}(-\mathbf{i}$ $\operatorname{Sen} \varphi+\operatorname{Cos} \varphi+\mathbf{j} \operatorname{Sen} \varphi \operatorname{Sen} \varphi+\mathbf{k} \operatorname{Cos} \theta)$ yacen en el plano $\mathrm{z}=0$, el ángulo polar $\theta$ es igual a 
$\pi / 2$. Además, como el radio de la espira está dado por a, el módulo del radio vector $r$ también es a, entonces

$$
\mathrm{d} \mathbf{s}=\mathrm{a}(-\mathbf{i} \operatorname{Sen} \varphi+\mathbf{j} \operatorname{Cos} \varphi) \mathrm{d} \phi
$$

Por tanto, la ecuación 2 se convierte en

$$
\mathrm{d} \mathbf{F}=\mathrm{I} \text { a } B_{0}(-\mathbf{i} \operatorname{Sen} \varphi+\mathbf{j} \operatorname{Cos} \varphi) \times\left(\mathbf{i} \operatorname{Sen} \theta_{0} \operatorname{Cos} \varphi_{0}+\mathbf{j} \operatorname{Sen} \theta_{0} \operatorname{Sen} \varphi_{0}+\mathbf{k} \operatorname{Cos} \theta_{0}\right) d \varphi
$$

Desarrollando el producto vectorial en la ecuación 3 y agrupando términos resulta

$$
\mathrm{d} \mathbf{F}=\mathrm{I} \text { a } \mathrm{B}_{0}\left[\mathbf{i} \operatorname{Cos} \theta_{0} \operatorname{Cos} \varphi+\mathbf{j} \operatorname{Cos} \theta_{0} \operatorname{Sen} \varphi-\mathbf{k} \operatorname{Sen} \theta_{0} \operatorname{Cos}\left(\varphi-\varphi_{0}\right)\right] \mathrm{d} \varphi
$$

Pero como el torque diferencial $\mathrm{d} \tau$ está dado por

$$
\mathrm{d} \tau=\mathbf{r} \times \mathrm{d} \mathbf{F}
$$

Se tiene entonces,

$$
\mathrm{d} \tau=\mathrm{I} \mathrm{a}^{2} \mathrm{~B}_{0}(\mathbf{i} \operatorname{Cos} \varphi+\mathbf{j} \operatorname{Sen} \varphi) \times\left[\mathbf{i} \operatorname{Cos} \theta_{0} \operatorname{Cos} \varphi+\mathbf{j} \operatorname{Cos} \theta_{0} \operatorname{Sen} \varphi-\mathbf{k} \operatorname{Sen} \theta_{0} \operatorname{Cos}\left(\varphi-\varphi_{0}\right)\right] \mathrm{d} \varphi
$$

Efectuando el producto vectorial en la ecuación 6 rinde

$$
\mathrm{d} \tau=\mathrm{I} \mathrm{a}^{2} \mathrm{~B}_{0} \operatorname{Sen} \theta_{0}\left[-\mathbf{i} \operatorname{Sen} \varphi \operatorname{Cos}\left(\varphi-\varphi_{0}\right)+\mathbf{j} \operatorname{Cos} \varphi \operatorname{Cos}\left(\varphi-\varphi_{0}\right)\right] \mathrm{d} \varphi
$$

El torque total se halla integrando el ángulo $\varphi$ en su dominio completo, es decir,

$$
\tau=\mathrm{I} \mathrm{a}^{2} \mathrm{~B}_{0} \operatorname{Sen} \theta_{0}\left[-\mathbf{i}_{0} \int 2 \pi \operatorname{Sen} \varphi \operatorname{Cos}\left(\varphi-\varphi_{0}\right) \mathrm{d} \varphi+\mathbf{j}_{0} \int 2 \pi \operatorname{Cos} \varphi \operatorname{Cos}\left(\varphi-\varphi_{0}\right) \mathrm{d} \varphi\right]
$$

Llevando a cabo las integrales en la ecuación 8 rinde

$$
\tau=\mathrm{I} \mathrm{a}^{2} \mathrm{~B}_{0} \operatorname{Sen} \theta_{0}\left(-\mathbf{i} \pi \operatorname{Sen} \varphi_{0}+\mathbf{j} \pi \operatorname{Cos} \varphi_{0}\right)
$$

Factorizando $\pi$ y reinsertando $\mathrm{B}_{0} \operatorname{Sen} \theta_{0}$ en la ecuación próxima anterior causa

$$
\tau=\mu\left(-\mathbf{i} B_{0} \operatorname{Sen} \theta_{0} \operatorname{Sen} \varphi_{0}+\mathbf{j} B_{0} \operatorname{Sen} \theta_{0} \operatorname{Cos} \varphi_{0}\right)
$$

Expresando cada vector unitario Cartesiano en la ecuación 10 como un producto vectorial da

$$
\tau=\mu\left[(\mathbf{k} \times \mathbf{j}) \mathrm{B}_{0} \operatorname{Sen} \theta_{0} \operatorname{Sen} \varphi_{0}+(\mathbf{k} \times \mathbf{i}) \mathrm{B}_{0} \operatorname{Sen} \theta_{0} \operatorname{Cos} \varphi_{0}\right]
$$

Pre - factorizando el vector unitario $\mathbf{k}$ en la ecuación 11 genera

$$
\tau=\mu \mathbf{k} \times\left(\mathbf{i} \mathrm{B}_{0} \operatorname{Sen} \theta_{0} \operatorname{Cos} \varphi_{0}+\mathbf{j} \mathrm{B}_{0} \operatorname{Sen} \theta_{0} \operatorname{Sen} \varphi_{0}\right)
$$

Como el producto vectorial de dos vectores paralelos o antiparalelos es nulo se hace conveniente y oportuno agregar el término $\mathbf{k} \operatorname{Cos} \theta_{0}$ al vector entre paréntesis de la ecuación 12 así

$$
\tau=\mu \mathbf{k} \times\left(\mathbf{i} \mathrm{B}_{0} \operatorname{Sen} \theta_{0} \operatorname{Cos} \varphi_{0}+\mathbf{j} \mathrm{B}_{0} \operatorname{Sen} \theta_{0} \operatorname{Sen} \varphi_{0}+\mathbf{k} \operatorname{Cos} \theta_{0}\right)
$$

Por inspección directa de la ecuación (13) se desprende que

$$
\tau=\mu \times \mathbf{B}_{0}
$$


La expresión escalar correspondiente a los módulos de la ecuación 14 viene dada por

$$
\begin{aligned}
& \tau=\mu \mathrm{B}_{0} \operatorname{Sen}\left[\operatorname{Cos}^{-1}\left(\mu \bullet \mathbf{B}_{\mathbf{0}}\right)\right] \\
& \text { O sea, } \quad \tau=\mu \mathrm{B}_{0} \operatorname{Sen} \theta_{0}
\end{aligned}
$$

El elemento diferencial de energía potencial magnética, $\mathrm{dV}_{\mathrm{m}}$, está dado por

$$
d V_{m}=\mu B_{0} \operatorname{Sen} \theta_{0} d \theta
$$

Es conveniente que $\mathrm{V}_{\mathrm{m}}$ adopte el valor 0 para $\theta=\pi / 2$ así que

$$
\mathrm{V}_{\mathrm{m}}=-\mu \mathrm{B}_{0} \operatorname{Cos} \theta_{0 \pi / 2}{ }^{\theta 0}
$$

Evaluando los límites impuestos en la ecuación 17 se obtiene

$$
\mathrm{V}_{\mathrm{m}}=-\mu \mathrm{B}_{0} \operatorname{Cos} \theta_{0}
$$

Como el módulo y la dirección del campo magnético uniforme han supuestos arbitrarios, no hay inconveniente alguno en cambiar las variables $\mathrm{B}_{0}$ y $\theta_{0}$ por $\mathrm{B}$ y $\theta$, entonces

$$
\mathrm{V}_{\mathrm{m}}=-\mu \mathrm{B} \operatorname{Cos} \theta
$$

Un electrón, con carga eléctrica - e y masa $m$, que efectúa $v$ revoluciones por segundo en torno al núcleo atómico, en una órbita circular de radio a, porta un momento magnético $-\mathrm{ev} \pi \mathrm{a}^{2}$, pero como su velocidad lineal es $2 \pi v a$, su cantidad de movimiento angular L es $2 \pi \mathrm{mva}{ }^{2}$ así que el momento magnético del electrón, expresado en forma vectorial, es

$$
\mu=-[\mathrm{e} /(2 \mathrm{~m})] \mathbf{L}
$$

La energía potencial magnética puede ser entonces expresada como

$$
\mathrm{V}_{\mathrm{m}}=[\mathrm{e} /(2 \mathrm{~m})] \mathrm{L} B \operatorname{Cos} \theta
$$

El número cuántico magnético $\mathrm{m}_{\ell}$ determina parcialmente la dirección de $\mathbf{L}$ al especificar que la componente $\mathrm{L}_{\mathrm{z}}$ a lo largo del campo magnético es

$$
\mathrm{L}_{\mathrm{z}}=\mathrm{m}_{\ell} \hbar
$$

En la ecuación 22 h es la constante de Planck h, dividida por $2 \pi$.

La energía magnética de un átomo con número cuántico magnético $\mathrm{m}_{\ell}$, es pues

$$
\mathrm{V}_{\mathrm{m}}=\mathrm{m}_{\ell}[\mathrm{e} \hbar /(2 \mathrm{~m})] \mathrm{B}
$$

La expresión [e ћ / (2m)], contenida en la ecuación 23, es conocida como el magnetón de Bohr; cabe anotar que tanto Niels Bohr como Max Planck fueron insignes físicos.

Frente a un campo magnético externo, la energía de un estado atómico depende, en gran medida, del número cuántico principal n que determina y cuantiza la energía del estado atómico y, en menor medida, de $\mathrm{m}_{\ell}$ por lo que la disgrega en subestados con valores ligeramente distintos al de la energía en ausencia del campo. Este desdoblamiento de las líneas espectrales es llamado efecto Zeeman normal. En el caso del átomo de hidrógeno, $\mathrm{m}_{\ell}$ solo puede adoptar los valores 0 y \pm 1 de modo que, en vez de la línea correspondiente a la frecuencia $v_{0}$, aparecen tres:

$v_{1}=v_{0}-e B /(4 \pi m), v_{0}, v_{2}=v_{0}+e B /(4 \pi m)$

Constituye un ejercicio muy interesante el cálculo del campo magnético en el marco de referencia del electrón, es decir, considerando que es el núcleo atómico el que gira alrededor del electrón. Conviene recordar que el campo magnético B producido al centro de una espira circular de radio a, en un ambiente con permeabilidad magnética $\mu_{0}$, que porta una corriente I (Davies, 1965), está dado por:

$$
\mathrm{B}=\mu_{0} \mathrm{I} /(2 \mathrm{a})
$$

El núcleo atómico, con carga eléctrica e, al efectuar $v$ revoluciones por segundo en torno al electrón, en una órbita circular de radio a, representa la corriente I dada por

$$
\mathrm{I}=\mathrm{ev}
$$


Entonces, reemplazando el valor de I, dado por la ecuación 26, en la ecuación (25) da

$$
\mathrm{B}=\mu_{0} \mathrm{ev} /(2 \mathrm{a})
$$

En el átomo de Bohr, en estado fundamental, la frecuencia $v$ y el radio vienen $d a-$ dos por

$v=\mathrm{e} /\left[2 \pi\left(4 \pi \varepsilon_{0} \mathrm{~m} \mathrm{a}^{3}\right)^{1 / 2}, \mathrm{a}=\mathrm{h}^{2} \varepsilon_{0} /(\pi \mathrm{m} \mathrm{e}) \quad(28 \mathrm{a}, \mathrm{b})\right.$

Sustituyendo las ecuaciones 28 en la ecuación (27) produce

$$
\mathrm{B}=\pi \mu_{0} \mathrm{~m}^{2} \mathrm{e}^{7} /\left(8 \varepsilon_{0}{ }^{3} \mathrm{~h}^{5}\right)
$$

Reemplazando valores numéricos en la ecuación 29 rinde

$$
\mathrm{B}-13 T
$$

Este campo magnético es muy fuerte; habida cuenta que el valor máximo del campo magnético sobre la superficie de la Tierra se da en sus polos Magnéticos, Norte y Sur, y es del orden de apenas $6 \mu T$ (Plonsey and Collin, 1961).

\section{Conclusión}

Se ha logrado un exitoso tratamiento general del torque experimentado por un dipolo magnético frente a la presencia de un campo magnético uniforme arbitrario a diferencia de los enfoques ad hoc existentes en las referencias pertinentes.

\section{Recomendación}

Es oportuna y conveniente la continuación de esta línea de investigación para explicar de manera clara y precisa el efecto Stern - Gerlach consistente en la verificación experimental de la separación de los dipolos magnéticos de signo opuesto al pasar a través de un campo magnético homogéneo.

\section{Referencias}

Beiser, A. (1988). Conceptos de Física Moderna, McGraw-Hill. México, pp. 135, 189-191, 211.

Davies, K. (1965). Ionospheric Radio Propagation, National Bureau of Standards Monograph 80, U. S. Government Printing Office. Washington D.C., pp. 23-24.
Plonsey, R. and Collin, R. E. (1961). Principles and Applications of Electromagnetic Field, International Student Edition, McGraw Hill Book Company, Inc., Kōgakusha Company, Ltd. Tokyo. pp. 208-211.

\section{Agradecimiento}

El autor expresa su agradecimiento a las autoridades de la Unversidad de San Martín de Porres, señores José Antonio Chang Escobedo, Raúl Bao García, Manuel Cáceres Lampen, Andrés Ramos Salas, Jaime Palacios Olivos y Jorge Tejada Polo por fomentar, proactivamente, las tareas de investigación, en general, y de este artículo, en particular. 
